\title{
Article
}

\section{Collaborative service delivery to address public health issues within a musculoskeletal setting: evaluation of the Healthy Mind, Healthy Body project}

Holehouse, Kelly, Oliver, Karen, Rawlinson, Gillian and Roddam, Hazel

Available at http://clok.uclan.ac.uk/26049/

Holehouse, Kelly, Oliver, Karen, Rawlinson, Gillian and Roddam, Hazel ORCID: 0000-0002-0637-1801 (2019) Collaborative service delivery to address public health issues within a musculoskeletal setting: evaluation of the Healthy Mind, Healthy Body project. International Journal of Therapy and Rehabilitation, 26 (11). pp. 1-15. ISSN 1741-1645

It is advisable to refer to the publisher's version if you intend to cite from the work.

For more information about UCLan's research in this area go to http://www.uclan.ac.uk/researchgroups/ and search for < name of research Group>.

For information about Research generally at UCLan please go to http://www.uclan.ac.uk/research/

All outputs in CLoK are protected by Intellectual Property Rights law, including Copyright law. Copyright, IPR and Moral Rights for the works on this site are retained by the individual authors and/or other copyright owners. Terms and conditions for use of this material are defined in the policies page. 
Collaborative cross agency service delivery to address public health issues within an MSK setting: evaluation of the Healthy Mind, Health Body project

\section{Short title: HMHB}

Kelly Holehouse ${ }^{1}$,

Karen Oliver ${ }^{1}$,

Gillian Rawlinson²,

Hazel Roddam ${ }^{2}$

${ }^{1}$ East Lancashire Hospitals NHS Trust

${ }^{2}$ School of Health Sciences, University of Central Lancashire

Corresponding author: Hazel Roddam, HRoddam@uclan.ac.uk, 01772895484

\section{Acknowledgements}

The authors wish to express their thanks to all the patients who completed the HMHB feedback survey. Also the Physiotherapy Team at Barbara Castle Way Health Centre, the Integrated Musculoskeletal Pain and Rheumatology Service (IMPReS) at East Lancashire NHS Trust, Mindmatters (Lancashire Care NHS Foundation Trust), The Wellbeing Service (Blackburn with Darwen) and our patient representative Geraldine.

\section{Funding Source}

This project was supported in part by a bursary awarded by the Cumbria and Lancashire CAHPR (Council for Allied Health Professions Research) Hub.

All authors confirm that they have no conflict of interest in this paper. Approval for this service evaluation was given by the host health organisation. 


\section{Abstract}

Background: There is a need for a greater focus on public health and its impact on MSK conditions within healthcare delivery and physiotherapists are well positioned to support this. Outpatient Physiotherapy Musculoskeletal (MSK) services traditionally focus on rehabilitation and physical exercise yet many service users require support to improve both their mental and physical health. Aims: This innovative service improvement aimed to embed integrated health promotion within MSK physiotherapy service delivery. Method: A physiotherapy-led multi-professional team introduced patients to other community-based support services to address wider health needs. Findings: Service evaluation demonstrated a high uptake of self-referral to those community services, validating the potential benefit for management of MSK conditions. Positive patient feedback indicates that patients valued the service and were well supported to engage with health improvement. Conclusions: MSK Physiotherapy services need to consider the wider aspects of health putting public health at the heart of MSK service delivery.

\section{Key messages}

- Both physical and mental health promotion can be effectively incorporated into the physiotherapy management of individuals with MSK conditions

- Clear and effective signposting of patients can result in a higher uptake of community based wellbeing services

- Positive patient feedback indicates the high level of value placed on collaborative multidisciplinary management and support. 


\section{Reflective Questions}

- What consultations have you undertaken to understand the priority concerns and wellbeing challenges of your local patient populations?

- How do your local physiotherapy musculoskeletal services address patients' motivation and promote their mental health and wellbeing?

- How effectively do you signpost your patients to local community based wellbeing services?

- How might you evaluate the effectiveness of public health initiatives to increase patients' active engagement in healthier lifestyles?

\section{Background}

The UK National Health Service (NHS) Five Year Forward View (2017) outlines the need for a radical upgrade in prevention and public health, enabling people using health services to have greater control of their own care, and for the NHS to better integrate care delivery. Blackburn with Darwen is one of the $20 \%$ most deprived districts and unitary authorities in England, where estimated levels of adult excess weight, smoking, physical inactivity and mental health issues are worse than the England average (Public Health England 2017). MSK disorders are recognised as a long term condition and it is widely acknowledged their impact on other co-morbidities including Diabetes, Obesity, Heart Disease, Chronic kidney disease and Depression. (Richmond Group 2018). The burden to the NHS and Social care of long term conditions is $f 7$ in every $f 10$ of the Health and social Care budget spent. (DoH, 2012). Those living with long term conditions use 6 times more GP 
consultations and attend Accident and Emergency departments 5 times more frequently. (Richmond Group 2018). Addressing modifiable risk factors such as Physical Inactivity, Obesity, Smoking and MSK injury at every opportunity can influence many associated co-morbidities in a clinically and cost effective manner. Current systems and services, across the public and voluntary sector, are largely geared around single diseases and focusing on individual diseases devalues the cumulative impact on individual health and wellbeing and the wider impact on individual's lives. Professor Sir Michael Marmot (Fair Society, Healthy Lives 2012) highlighted the requirement to tackle the wider social determinants of health. It is also acknowledged that a 'one size fits all' approach is not helpful when public health challenges vary from one neighbourhood to another. (Healthy Lives, Healthy People: Our Strategy for Public Health in England 2010). To meet these new health challenges we need transformation in services and practice, both nationally and internationally. Current fragmented approaches to mental and physical health care occur within primary care and may lead to missed opportunities to provide quality, effective and efficient care (The Kings Fund and Centre of Mental Health 2012). It is suggested that closer working and better integration of mental health specialists with other primary care health professionals will improve the quality of care delivery (The NHS Five Year Forward View, 2017). Allied Health Professionals (AHPs) including physiotherapists, have a key role to play in supporting the health and wellbeing of patients, carers, families and communities (PHE \& Allied Health Professions Federation 2015).

The patient population attending the Integrated Musculoskeletal, Pain and Rheumatology Service (IMPReS) service at Barbara Castle Way Health Centre with musculoskeletal conditions, demonstrates cultural and ethnic diversity, a wide socio economic range, psycho social issues, along with multiple physical problems and co-morbidities. It is recognised that a high proportion of patients presenting to physiotherapy-led teams with back pain display high anxiety levels which is widely documented as a potential barrier to rehabilitation, as categorised by the validated STARTback tool (Hill et al 2008, Hassett et al 2016, NICE 2016). The importance of identifying poor 
prognostic factors in MSK presentation at an early stage are well documented, as is the importance of being able to deliver matched treatments to tackle these in order to achieve optimal outcomes (Hill et al 2011, Chou et al 2007). STARTback recommends a combined physical and cognitive behavioural approach for those patients categorised as 'high risk' in a bid to reduce pain and disability and improve psychological functioning (Hill et al 2008). Regular physical activity not only benefits MSK conditions, maintaining healthy weight, improving bone strength, balance and coordination but is also shown to have beneficial effects on reducing pain (Skou et al 2018) and wider physical health, as well as improving the sense of well-being, lowering anxiety and depressive symptoms (Anderson 2013, NICE 2016, DOH 2011). Public Health England (PHE 2013) reported that low levels of physical activity could lead to premature deaths. It was estimated that physical inactivity alone could potentially cost $f 0.9$ billion based on the occurrences of diseases. (Scarborough et al 2011). Addressing the wider modifiable public health risk factors will impact on both MSK and other co-morbidities such as cardiovascular disease, hypertension, diabetes, cancer and, fragility fractures (ARUK 2014). Exercise referral schemes seeking to increase physical activity levels have a range of positive health and well-being effects for those patients who are relatively inactive, lead sedentary lifestyles, and have existing physical and mental health issues that put them at risk of ill health. Specific recommendations already exist with regards to this for those patients presenting with Lower Back Pain and Depression (NICE 2014). This guidance also recommends that health professionals carry out an assessment for patients with suspected anxiety disorders and refer to a practitioner who is trained to carry out a more comprehensive assessment and provide appropriate input via an integrated approach alongside a physical activity programme where appropriate.

With an increased number of complex patients being referred to the IMPReS service there was acknowledgement that a holistic management plan is needed in order to achieve optimal health and treatment outcomes, with a recognised need "to do things differently" (All our Health 2016). Collaborative working with local community providers offers opportunities to address the 
HMHB

management of these patients and achieve long-term health improvements and has been identified as a key priority of The Accountable Health and Care Partnership for Pennine Lancashire in the 'Draft Pennine Plan' (2017).

\section{Methods}

The Six Stage project management approach (NHSI ACT Academy 2017) was used in the design of this project. Initially the area of improvement was identified by the clinical team and supported by local demographic data. Two clinician led mini focus group were held. The first consisted of four patients recruited from an active clinical caseload. The second group consisted of 6 clinicians. Both sessions lasted 30 - 40minutes. The aim was to gain stakeholder analysis including a mapping exercise to identify relevant local services and agencies. Following a review of existing pathways and clinician skill sets, actions were identified to improve quality and holistic management of the MSK patient with co-morbidities. A communication training programme and mentorship system for all clinicians was initiated. Two key services were identified. Firstly The Wellbeing service, a community based service designed to support and improve the health of the local population. This service offers help and support on activity levels, smoking cessation, weight loss, alcohol withdrawal, and sleep hygiene. Secondly The Mindsmatter service which is a community based wellbeing service providing support and treatment for a variety of mental health problems such as depression and anxiety. They offer a range of supports to help people make positive changes to reduce stress and anxiety and improve wellbeing. These include stress control classes, wellbeing workshops, supported online CBT programmes, one to one and telephone support.

A patient information leaflet was compiled as an initial resource to increase awareness and referral to the available Wellbeing and Mindsmatter services. However, this single intervention approach was insufficient and there was still a group of patients who failed to engage with services offered. A 
HMHB

more seamless transitional process to the community sector was required between the physiotherapy team and these two community services.

In 2016 a new service "Healthy Mind, Healthy Body" (HMHB) was launched within the IMPReS service. This comprised a monthly 90 minute physio-led multi-professional session for patients; including a patient representative who provided a detailed narrative of their own experience of these local services. Patients from the IMPReS service were invited based on STARTback categorisation (High/Medium) (Hill et al 2008), informal assessment findings and shared patient and clinician decision making. Exclusion criteria were minimal, with the aim of reaching as wide a population as possible given the well documented benefits of public health interventions. The only definitive requirement was the ability to understand English. The HMHB session could be accessed at any stage of the patient's physiotherapy journey, allowing this provision to be responsive to meet patients' need.

Patient feedback was elicited through a locally designed questionnaire after the HMHB session. This had been developed by the physiotherapy team together with the patient representative and comprised a mixture of closed, multiple-choice and open questions. The questions included patients' intentions for future actions related to their own lifestyle and wellbeing. A content analysis was undertaken to generate themes (Nowell et al 2017) that will be used to inform further refinements of the HMHB initiative.

\section{Results}

A total of 147 patients attended a HMHB session over 12 months (December 2016 - November 2017 inclusive). Of all the patients referred to a HMHB session, $77 \%$ attended. Over $90 \%$ of the patients attending a HMHB session opted into one or both of the further long term wellbeing service options. 
HMHB

The evaluation data presented in Figure 1 show that $82 \%$ of patients initiated a self-referral to the Wellbeing service as a result of attending the HMHB intervention; and $56 \%$ of those went on to complete the referral process to the Wellbeing service. In addition, $32 \%$ of patients initiated a selfreferral to the Mindsmatter service as a direct result of their attendance at the HMHB session; and $81 \%$ of these accessed the Mindsmatter service.

(Figure 1 here)

Self-reported patient feedback was collected at the time each patient attended the HMHB session using our survey tool over a six month period (April 2017-September 2017). This demonstrated that 91\% of patients reported that they judged the session as 'useful' and 'quite useful' (Figure 2).

Eighty six percent of responders reported they would change their lifestyle; a further $14 \%$ stated they 'hopefully' would: however further follow-up work is needed to explore individuals' subsequent actions based on those intentions.

"the whole session opened my eyes to show anything is possible ... only willpower to make it happen".

(Figure 2 here)

Completed questionnaires were collected from 66 patients in 6 of the HMHB sessions. There were 47 separate responses to the open question about perceived value of attending the HMHB session, with a wide range of comments about what they had learned. Many patients expressed surprise at the amount of new information and advice they had gained through the session content. There was also a predominant acknowledgement that they had found the session delivery to be highly motivating and empowering, especially the presentations from former patients: 
HMHB

"Patient's experience - gaining information about a patient's real life situation and how she improved her health"

"Geraldine's talk about her journey - brilliant!"

The participants' feedback demonstrated the impact of the session, particularly the inclusion of the importance to consider mental wellbeing as well as physical health:

"All the information and link to mental health and physical health"

"Both aspects of the session were very useful as it gives you awareness of what is causing you problems"

For many participants, this was the first time they had received information about mental health services and they reported that they valued the way this was presented to defuse any stigma:

"Openness about mental health"

"All of the session was useful, made me realise some of the words e.g. depression and stress, and the physical side of things need I do need help with"

Many of the participants commented on how helpful they found the information relating to other relevant services and how they could access these:

"Hearing about options that can help me to be healthier".

"Telling us what is available us and wow - what a lot!"

The content analysis of the comments generated 14 basic descriptive codes, which were categorised into 8 organising codes and 3 global codes, which are shown in Table 1:

(Table 1 here)

The overall impact and perceived value of the HMHB session by this convenience sample of 66 patients is shown in Figure 3. The survey also included a question to elicit patients' perceptions of "the least 
HMHB

useful" aspect of the session. The answers to this exclusively commented on environmental aspects of the venue; none referred to either the content or delivery of the session.

(Figure 3 here)

\section{Discussion}

Physiotherapists are optimally placed to deliver public health messages and health promotion to a large population (PHE \& Allied Health Professions Federation 2015). This service redesign demonstrates the potential for AHPs to be integral in the public health arena, with opportunities to work collaboratively with other local wellbeing agencies and services. Referral into the HMHB session required both the clinician and patient to think holistically to explore links between their MSK concerns and their general health. The referral numbers into the session suggest that the communication training for clinicians had been well received and implemented. The attendance rate from physiotherapy referral to the HMHB session was relatively high (77\%), demonstrating a need within the local population for the wider aspects of health to be identified and addressed when dealing with a concurrent MSK problem; an approach that is well documented in the literature (All Our Health 2016, Royal Society for Public Health 2017).

The dropout rate of $23 \%$, from referral to attendance at the session may be due to multiple factors; potentially including a need for further training to improve clinicians' identification of appropriate patients that are likely to take up services offered. This may be improved with the production of a more detailed patient information leaflet preparing patients for this form of intervention and promoting their engagement as well utilising opportunities to explore patients' health behaviours and motivations during consultations. This increased signposting of patients to the HMHB session or to other community services supports the public health agenda to make every contact count (MECC) and to ensure a holistic treatment plan is implemented (Royal Society for Public Health 2017). 
HMHB

Greater patient involvement and co-production of future service refinements may also enhance attendance.

The high proportion of patients who went on to engage with these local services after attending a HMHB session suggests that the topics covered within the signposting session were relevant, and that those services were appropriate. Initial interest and uptake after attending HMHB session was significantly higher for the Wellbeing Service than Mindsmatter. The nature of mental health conditions may make it challenging for this cohort of patients to engage and could therefore explain this finding. Also, the broader scope of the Wellbeing Service may make this more appealing to a larger group of patients as reflected in the referral rates. Our results did not specifically identify which elements of the Wellbeing Service the patients accessed; though it could be anticipated that all attempts to improve their wellbeing may result in a beneficial outcome. Improvements in an individual's lifestyle and physical health may also improve mental health, despite the service not being specifically focussed on mental health support. Although $9 \%$ of patients did not initiate an immediate referral to either service, public health messages delivered during this intervention may have influenced their lifestyle choices or may start them on the pathway to better health. Behavioural change is complex and action may be initiated at a later stage and there were subsequent self-referrals to both services. The implicit benefits of preventative health messages should not be underestimated, although there are multiple methodological challenges to measure these in practice (Royal Society for Public Health 2017).

The dropout rate indicated by the difference between the number of patients who self-referred from the session and those who actually attended the Wellbeing service may indicate a need to further streamline the referral to contact process, as well as considering ways to offer further support in this transitional phase across the services. This is an issue which has some potential funding and staffing implications with the current pathway and warrants further exploration with 
the local service providers and public health commissioners. This highlights the difficultly in measuring the benefit of a single health intervention when dealing with long-term health conditions and supports the need for the delivery and recording of consistent health messaging at every contact (Good Public Health Practice Framework 2016).

One of the key principles of public health intervention is successfully engaging people in behavioural changes for health and wellbeing. This is widely acknowledged as being difficult to measure in the short term (Royal Society for Public Health 2017). Improvements in population health and wellbeing outcomes should be seen in coming years if we apply a broad and consistent public health approaches.

In line with the Public Health England framework (PHE 2018), this project demonstrates cross agency partnership working that utilises existing resources to deliver population based, holistic patient care in order to improve quality of life and address the wider aspects of health. The partnership with the community services offered the benefit of shared learning for all parties involved, integration of treatment plans and smooth pathway for patient transfer, and may have facilitated the attendance of patients to the community services. There is a recognised need to de-medicalise the treatment of many MSK conditions in order to achieve long-term health benefits and supported self-care of long term conditions. Supporting the appropriate transition away from medical care into the community sector in this way aims to support achieving this goal. Services require constant evaluation and improvement including input from service users. An immediate improvement of this project is the development of an Urdu speaking version in recognition of the local population demographics. Further analysis of our local population inequalities may highlight further areas for targeted improvement. No additional funding or resources were required in the implementation of this service redesign, providing excellent value for money and sustainability. As recommended in the Public Health Skills and Knowledge framework (2018), working with mental and physical wellbeing agencies in an innovative, collaborative approach has provided a new cost effective solution that 
HMHB

contributes to addressing the complex and broad issues of public health priorities. Community based physical and mental wellbeing schemes such as those involved in this pilot, exist across the United Kingdom and are accessible to health professionals, adding to the potential wider implementation and scale up and spread of this approach.

\section{Conclusion}

Physiotherapists have a role to play in public health and are committed to evaluating population need and reviewing evidence based practice to ensure the provision of quality, cost effective, patient-focused service delivery. Using cross agency partnerships to extend the scope of a service supports delivery of the Good Public Health Practice framework (2016) and supports more integration of health and community services. In this initiative, cross agency working has demonstrated beneficial upskilling of staff across all three service providers, development of strong links across services and improved communication. Service-users valued the new service and rates of self-referral to community groups were high. The ultimate benefactors of this are the service users who are now receiving a more holistic, seamless and rounded care plan aimed to improve overall health impacts. The foundations of public health interventions require not only a population based focus but also the engagement of individuals. Responding to patient feedback, as well as gathering and monitoring outcome data ensures this project continues to engage and empower all stakeholders to make lasting behavioural changes and long term health improvements.

Future work is required to further develop links with a wider range of community services to improve the health and wellbeing of the local population. Whilst this service is focussed on delivering public health messages to a subset of patients, a public health approach has also transferred into other aspects of physiotherapy care; supporting empowerment of patients and the promotion of sustainable health improvements. Further work is also required to determine specific 
HMHB

health inequalities within the local population. This pilot has demonstrated the benefits of utilising physiotherapists as a key part of the public health workforce in supporting delivery of health improvement interventions and is an example of innovative and cost-neutral service collaboration and integration.

\section{References}

1. NHS Improvement ACT Academy (2017) Transformational Change through System Leadership programme

2. Afton L. Hassett, Jenna Goesling, Sunjay N. Mathur, Stephanie E. Moser, Chad M. Brummett, Kimberly T. Sibille. (2016) Affect and Low Back Pain: More to Consider than the Influence of Negative Affect Alone Clin J Pain. 2016 Oct; 32(10): 907-914 doi:10.1097/AJP.0000000000000350

3. 'All our Health'. (2016). https://www.gov.uk/government/publications/all-our-health-aboutthe-framework

4. Anderson, E. Shivakumar, G. (2013). 'Effects of exercise and physical activity on anxiety'. Frontiers in Psychiatry. 4: 27.

5. Arthritis Research UK. Musculoskeletal Health - a public health approach (Internet). 2014 (cited 2015 January 19) https://www.arthritisresearchuk.org/policy-and-publicaffairs/policy-reports/musculoskeletal-health-a-public-health-approach.aspx 
6. Chou, R. Qaseem, A. Snow, V. Casey, D. Cross, JT Jr. Shekelle, P. Owens, DK. (2007). 'Diagnosis and treatment of low back pain: a joint clinical practice guideline from the American College of Physicians and the American Pain Society.' Clinical Efficacy Assessment Subcommittee of the American College of Physicians; American College of Physicians; American Pain Society Low Back Pain Guidelines Panel. Annals of International Medicine. Oct 2; Volume 147(Issue 7). Pages 478-91.

7. Department of Health, Physical Activity, Health Improvement and Protection. (2011) 'Start Active, Stay Active: A report on physical activity for health from the four home countries'

8. Department of Health (2012). Report. Long-term conditions compendium of Information: 3rd edition

9. 'Draft Pennine Plan'. (2017). Together a Healthier Future. Pennine Lancashire Accountable Health and Care Partnership. http://togetherahealthierfuture.org.uk/pennine-plan/

10. 'Fair Society, Healthy Lives: The Marmot Review'. Strategic review of health inequalities in England post-2010 (2012). Public Health. Volume 126, Supplement 1, 1 September 2012, Pages S4-S10

11. Good Public Health Practice Framework(2016) http://www.ukphr.org/wpcontent/uploads/2016/03/Good-Public-Health-Practice-Framework -2016 Final-2.pdf 
12. Hill, J. Whitehurst, D. Lewis,M. Bryan, S. Dunn, K. Foster, N. Konstantinou, K. Main, Mason, C. Somerville, S. Sowden, G. Vohora, K. Hay, E. (2011). 'Comparison of stratified primary care management for low back pain with current best practice (STarT Back): a randomised controlled trial'. The Lancet. Vol 378. Issue 9802. Pages 1560-1571.

13. Hill, J. Hay, E. Dunn, K. Lewis, M. Mason, E. Sowden, G. Somerville, S. Vohora, K. Whitehurst, D. Main, C. Konstantinou, K. (2008). 'A randomised clinical trial of subgrouping and targeted treatment for low back pain compared with best current care. The STarT Back Trial Study Protocol'. BMC Musculoskeletal Disorders. Vol 9:58

14. HM Government (2010) 'White Paper: Healthy Lives, Healthy People: our strategy for public health in England.'

15. National Institute for Health and Clinical Excellence (2016). Low back pain and sciatica in over 16s: assessment and management: NICE guideline NG59.

16. National Institute for Health and Clinical Excellence (2014). Physical activity: exercise referral schemes. NICE PH54.

17. Naylor, C. Parsonage, M. McDaid, D. Knapp, M. Fossey, M. Galea, A. (2012). 'Long-term conditions and mental health: The cost of co-morbidities'. The Kings Fund and Centre of Mental Health. ISBN 978185717 6339,Pages 32

18. Nowell LS, Norris JM, White DE, Moules NJ. (2017) Thematic Analysis: Striving to Meet the Trustworthiness Criteria. International Journal of Qualitative Methods https://doi.org/10.1177/1609406917733847 
19. Public Health England

http://fingertips.phe.org.uk/profile/health-profiles/area-search-

results/E12000002?search type=list-child-areas\&place name=North

20. Public Health Skills and Knowledge Framework(2018)

Https://www.gov.uk/government/publications/public-health-skills-and-knowledge-

framework-phskf/public-health-skills-and-knowledge-framework-phskf-update-march-2018

21. https://richmondgroupofcharities.org.uk/sites/default/files/final just one thing after anot her report - singles.pdf

22. Royal Society for Public Health (2017). 'Everyday Interactions: Measuring the Public Health Impact of Healthcare Professional'. https://www.rsph.org.uk

23. Scarborough, P. Bhatnagar, P. Allender. S. Foster, C. Rayner, M. (2011). 'The economic burden of ill health due to diet, physical inactivity, smoking, alcohol and obesity in the UK: an update to 2006-07 NHS costs'. Journal of Public Health, Volume 33, Issue 4, 1 December, Pages 527-535.

24. Søren Thorgaard Skou, Alessio Bricca, Ewa M. Roos (2018) The impact of physical activity level on the short- and long-term pain relief from supervised exercise therapy and education: A study of 12,796 Danish patients with knee osteoarthritis. Osteoarthritis and Cartilage 26 (11) 1474-1478 https://doi.org/10.1016/j.joca.2018.07.010 
HMHB

25. .'The NHS Five Year Forward View'. (2017) https://www.england.nhs.uk/five-year-forwardview 